\title{
Keeping surgeons in the loop: are handheld robotics the best path towards more autonomous actions? (A comparison of complete vs. handheld robotic hepatectomy for colorectal liver metastases)
}

\author{
Andrew A. Gumbs ${ }^{1}$, Mohammad Abu-Hilal ${ }^{2}$, Tzu-Jung Tsai ${ }^{3}$, Lee Starker ${ }^{4}$, Elie Chouillard ${ }^{5}$, Roland Croner $^{5}$ \\ 'Departement de Chirurgie Digestive, Centre Hospitalier Intercommunal de Poissy, Saint Germain-en-Laye 10, Rue du Champ \\ Gaillard, Poissy 78300, France. \\ ${ }^{2}$ Unità Chirurgia Epatobiliopancreatica, Robotica e Mininvasiva Fondazione Poliambulanza Istituto Ospedaliero, Brescia 25124, \\ Italy. \\ ${ }^{3}$ Koo Foundation Sun Yat-Sen Cancer Center, Taipei 00112, Taiwan. \\ ${ }^{4}$ Morristown Medical Center, Morristown, NJ 07960, USA. \\ ${ }^{5}$ Department of General-, Visceral-, Vascular- and Transplantation Surgery, University of Magdeburg, Magdeburg 39120, \\ Germany.
}

Correspondence to: Prof. Andrew A. Gumbs, Departement de Chirurgie Digestive, Centre Hospitalier Intercommunal de Poissy, Saint Germain-en-Laye 10, Rue du Champ Gaillard, Poissy 78300, France. E-mail: aagumbs@gmail.com

\begin{abstract}
How to cite this article: Gumbs AA, Abu-Hilal M, Tsai TJ, Starker L, Chouillard E, Croner R. Keeping surgeons in the loop: are handheld robotics the best path towards more autonomous actions? (A comparison of complete vs. handheld robotic hepatectomy for colorectal liver metastases). Art Int Surg 2021;1:38-51. https://dx.doi.org/10.20517/ais.2021.07
\end{abstract}

Received: 27 Aug 2021 First Decision: 18 Oct 2021 Revised: 21 Oct 2021 Accepted: 24 Nov 2021 Published: 1 Dec 2021

Academic Editors: W. Konrad Karcz, Takeaki Ishizawa Copy Editor: Yue-Yue Zhang Production Editor: Yue-Yue Zhang

\begin{abstract}
Aim: Some surgeons have been using some form of handheld robotics (HR) since liver resections began being done minimally invasively (MI); however, with the development of the complete robotic surgical systems (CRSS), they have lived in limbo neither being truly laparoscopic nor robotic. While doing the Study: International and Multicentered on Minimally Invasive Liver Resections for colorectal metastases (CRLM), we decided to evaluate these two different degrees of robotics, specifically the HR group and the group undergoing a completely robotic (CR) approach.
\end{abstract}

Methods: Four international centers (one in France, one in Germany, one in Taiwan, and one in the United States of America) were asked to join a retrospective review of cases to compare short- and long-term outcomes after open, laparoscopic, and robotic liver resection for CRLMs. For this study, only patients who had either HR or CR liver 
resections were included. HR was defined as cases done with a robotically controlled camera holder and a powered stapling device. Only patients with $\leq 3$ tumors that were $\leq 5 \mathrm{~cm}$ were included so that the preoperative characteristics of the two groups would be similar.

Results: In total, three centers did CR for CRLM (28 patients) and one center used HR (49 patients). MI resections were possible in $92.5 \%$ of patients when HR was used compared to $34.2 \%$ (22.6\% laparoscopic, $11.5 \%$ CR) when centers used CRSS. Mean operating room times were significantly longer after CR compared to HR resections, $234 \mathrm{~min}$ vs. $290 \mathrm{~min}(P=0.04)$. The morbidity and mortality rates were $4.1 \%$ and $2 \%$ after HR compared to $10.7 \%$ and $0 \%(P=N S)$, respectively. RO resection rates and resection margins were $100 \%$ and $9.6 \mathrm{~mm}$ compared to $89.3 \%$ and $6.7 \mathrm{~mm}$ after $\mathrm{HR}$ vs. $C R$, respectively $(P<0.05)$. The estimated blood loss and length of stay were similar in both groups, $264 \mathrm{~mL}$ and 4.9 days vs. $227 \mathrm{~mL}$ and 6.8 days $(P>0.05)$. Overall and disease-free survival at one and two years were $100 \%$ and $89.4 \%$ after HR vs. $100 \%$ and $72.9 \%$ after CR $(P>0.05)$.

Conclusion: HR and CR liver resections have similar short- and long-term outcomes; however, when HR is used, over $90 \%$ of cases can be done MI compared to under 35\% with CR. The added benefit of haptics and the ability for the operating surgeon to use hand assistance may account for this discrepancy. CRSS do not use haptics and surgeons must rely on visual cues; as robots become more autonomous, it may make more sense for computer engineers to work on the robot perceiving feedback and not the surgeon. HR may be the safest way to develop more autonomous actions in surgery and may yield the most benefits for patients by keeping the surgeon in the loop.

Keywords: Minimally invasive surgery, laparoscopy, liver resection, hepatectomy, robotic-assisted surgery, hepatic surgery, hepatobiliary, handheld robotics, complete surgical robotic system

\section{INTRODUCTION}

As early as 2002, surgeons began using complete robotic surgical systems (CRSS) for hepatectomy ${ }^{[1,2]}$. At around this time, surgeons divided into minimally invasive (MI) liver surgeons using standard laparoscopy, laparoscopy with handheld robotics (HR), or CRSS. The debate has continued as to whether robotic surgery should simply be considered a form of laparoscopy, or if it is a distinct technical entity ${ }^{[3]}$. Interestingly, the surgeons using HR were simply lumped into the laparoscopic group and excluded from the robotic one ${ }^{[4,5]}$.

Liver resections have increasingly been done with MI techniques. In fact, since Gagner ${ }^{[6]}$ performed the first laparoscopic liver resection in 1992, laparoscopy has become the gold standard for minor hepatectomies, mainly left-sided ${ }^{[6-9]}$. As experience grew, MI surgeons began performing major hepatectomies, including even those requiring the resection of neighboring organs and complex biliary reconstructions ${ }^{[10-18]}$. At the last consensus meeting held in Southampton in 2017, MI approaches were considered valid approaches for both minor and major hepatectomies ${ }^{[19]}$; moreover, the guidelines confirmed the oncological appropriateness of minimally invasive surgery in the management of both primary (especially in cirrhotic patients) and secondary lesions.

Just over 10 years after the first laparoscopic hepatectomy, surgeons began doing liver resections with CRSS (da Vinci, Intuitive Surgical, Sunnyvale, CA ${ }^{[1,2]}$. Ultimately, when larger robotic companies purchased the rights of smaller robots, other companies came onto the market with even smaller handheld robotic devices that also enabled some robotically controlled manipulation ${ }^{[20-22]}$, and even created HR surgical forceps and dissectors $^{[23]}$. Over the last 15 years, handheld powered robotic stapling devices fitted with sensors have also been developed and used ${ }^{[24]}$. Despite varying degrees of robotic utilization among MI surgeons, currently, only patients using complete robotic (CR) platforms are considered as robotic cases. This is unfortunate, 
because this bias has pigeon-holed surgeons and engineers to focus only on CRSS and not truly explore all the potential benefits of smaller and more affordable handheld robots ${ }^{[3]}$.

Multiple studies have shown clear advantages for patients when liver resections can be done MI. A recent meta-analysis of 2 randomized-controlled trials and 13 propensity score matched (PSM) trials even showed evidence suggesting a survival advantage for patients undergoing laparoscopic resection for colorectal liver metastases when compared to open resections ${ }^{[25]}$. However, the fact remains that the majority of hepatectomies are still done via open techniques at most, even specialized, centers. Although it is tempting to believe that there are indeed short- and long-term benefits to patients who are resected MI, the multiple potential problems with PSM, at the very least, raise the alarm that a significant degree of patient selection bias may be occurring when open is compared to MI hepatectomy ${ }^{[26]}$.

Currently, most research now focuses on comparing which MI approach to liver resection is superior, laparoscopic or robotic, with very little research being done on how to increase the number of patients who can actually undergo MI liver resection, with one of the main differences between the two techniques being the lack of haptics during CR surgery. Despite the rise of artificial intelligence (AI) and the dream of autonomous surgery, it is unclear as to the ultimate value of haptics ${ }^{[27]}$. CRSS do not offer haptics and robotic surgeons must rely on visual clues. Theoretically, autonomous surgery would not require haptics as the surgical maneuvers would be autonomous and done via the robot and a computer interface. Certain CRSS are already able to offer haptics; however, the augmentation of surgeon's resting tremor makes the haptics useless, and this function is currently not activated on commercially available robots ${ }^{[28]}$.

If the majority of liver resections are still being done via open techniques, which inherently has the best haptics, the question must be asked: why are more handheld robotic instruments that might be able to offer superior haptics not being increasingly utilized and developed? In an attempt to shed some light on this issue, we compared the results of a center using HR devices, specifically a robotically controlled laparoscope holder [Figure 1] and robotic gastrointestinal anastomosis (GIA) vascular staplers (Signia, Medtronic, Dublin, Ireland) [Figure 2], with those of groups using CR systems.

\section{METHODS}

We initiated a retrospective international multicenter study (Study: International Multi-center Minimally Invasive Liver Resection) analyzing outcomes after liver resection for colorectal liver metastases (CRLM). The primary endpoint was to assess the preoperative results between open and laparoscopic liver resection, and the results of that study will be reported elsewhere. Secondary endpoints included survival analysis. In this study, we compared centers that do hepatectomies using the CR vs. HR approach to see if any differences could be noted between these two varying degrees of robotics. In an effort to compare similar patients, only patients with $\leq 3$ tumors and with no tumor measuring more than $5 \mathrm{~cm}$ were included in the final analysis.

Patients' characteristics, operative parameters, and follow-up outcomes were regularly updated. Indications for surgery included both benign and malignant disease, either primary or secondary. Contraindications to laparoscopy include closed angle glaucoma, intracranial hypertension, and severe lung disease. Because a robotically controlled laparoscope holder (Video endosKopY, Endocontrol, Grenoble, France) [Figure 1] and a powered handheld stapler with sensing technology [Figure 2] were used, the procedures were considered $\mathrm{HR}^{[21]}$. The term CR was reserved for procedures done with a CRSS such as the da Vinci (Intuitive Surgical, Inc., Sunnyvale, CA, USA) or Versius robots (Versius Robotics, CMR, Cambridge, UK). The technique of HR liver resection with the robotically controlled laparoscope holder ${ }^{[18,22,29-32]}$ and the use of 


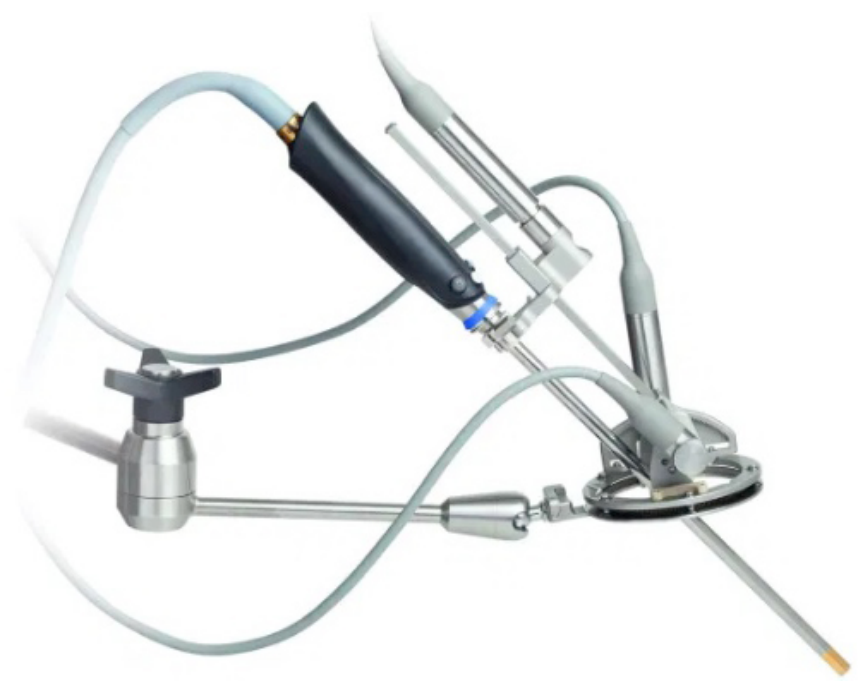

Figure 1. Video-endosKopy (ViKY), robotically controlled handheld laparoscope holder (ViKY, Endocontrol, Grenoble, France).

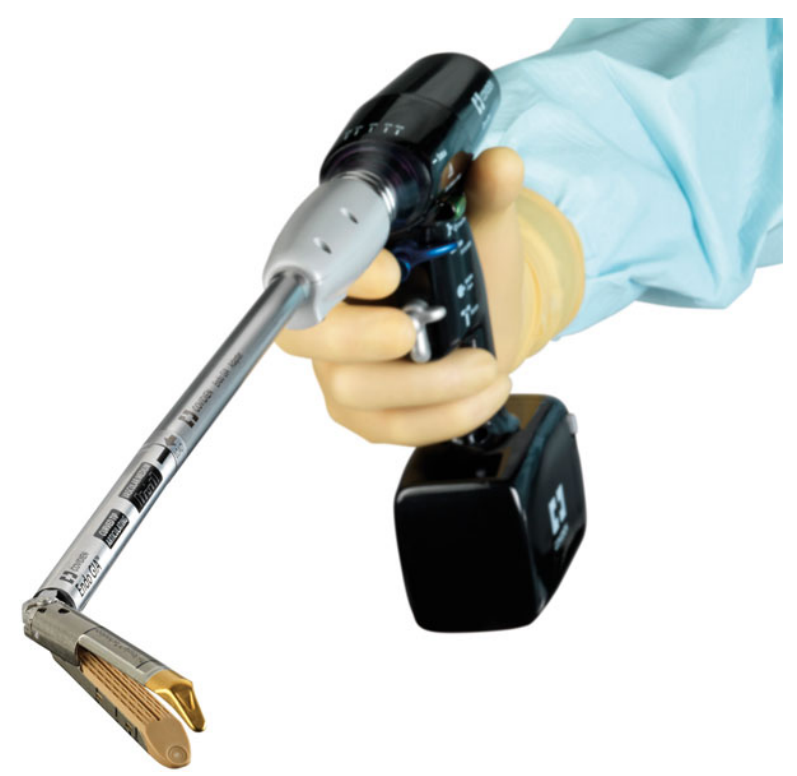

Figure 2. Handheld powered gastrointestinal stapler (Signia, Medtronic, Dublin, Ireland).

the powered vascular GIA stapler for liver resection was previously described ${ }^{[1,33]}$.

The analysis was undertaken on an intention to treat basis. The data that support the findings of this study are available from the corresponding author upon reasonable request. The main endpoint of the study was the postoperative mortality and morbidity of all patients who had MI in the HR group as compared to liver resections done by the CR group. Patients done with hand assistance were also considered to be done minimally invasively. Secondary endpoints included comparison of operative parameters, length of hospital stay, and long-term survival. The matching criteria included age, sex, benign or malignant disease, and Anesthesiology Society of America status score. Written informed consent was obtained from all patients. 
Preoperative embolization was obtained when an extended major hepatectomy was planned. Minor hepatectomy was defined as $<3$ hepatic segments, major hepatectomy was defined as $\geq 3$ hepatic segments but $\leq 4$ hepatic segments, and extended hepatectomy was defined as $\geq 4$ hepatic segments. Central hepatectomy was defined as the removal of hepatic segments 4, 5, and 8. Laparoscopic minor hepatectomy was usually performed via 3-4 trocars. Major and extended hepatectomies were performed with 5-6 trocars, usually $<1$ handbreadth below the right costal margin. Parenchymal transection was done with energy devices and hemostasis was achieved with the laparoscopic bipolar device. Named vessels were transected with the laparoscopic vascular linear GIA stapler. The Dindo-Clavien classification system was used with major complications defined as greater than Grade $2^{[34]}$.

Data analysis was performed using Social Science Statistics software (www.socscistatistics.com), and Prism 8: GraphPad software (https://www.graphpad.com/scientific-software/prism/) was used to generate KaplanMeier curves. Categorical data are expressed as percentages and quantitative data are presented as mean \pm standard deviation with median and range. Data were analyzed by using the Mann-Whitney $U$-test for continuous variables and the Fisher exact test for categorical values. The Mantel-Cox test was used for the survival curves. Statistical significance was defined as $P<0.05$.

\section{RESULTS}

From 2006 to 2019 at four international sites (in Germany, France, Taiwan, and the United States of America), liver resection for CRLM were done in a total of 430 patients. After exclusion criteria (size $<5 \mathrm{~cm}$ and 1-3 tumors) were met, the liver resections done for CRLM dropped to 289 (67.42\%) patients via laparotomy, 65 (15.1\%) patients via laparoscopy, 57 (13.3\%) using HR, and $36(8.4 \%)$ patients using CRSS. From November 2016 to September 2019, 28 hepatectomies meeting the exclusion criteria were done using CRSS at three different international centers (Germany, 12 patients; Taiwan, 9 patients; USA, 7 patients), and they were considered in the CR group. All three CR groups used the da Vinci robot (da Vinci, Intuitive Surgical, Sunnyvale, CA, USA). From November 2008 to April 2018, at the HR center, 63 liver resections were done for CRLM, of which 57 (90.1\%) were done minimally invasively. Of these, 49 hepatectomies meeting exclusion criteria (size $<5 \mathrm{~cm}$ and 1-3 tumors) were identified, which were considered the HR group.

In total, $92.5 \%$ (49 patients) of liver resections for CRLM were done using robotic assistance and 7.5\% (4 patients) were done via open techniques in the HR group. Of these, four (8.2\%) patients required hand assistance [Table 1]. At the CR centers, 34.2\% (83 patients) of liver resections were done MI over the respective time periods, specifically $11.5 \%$ robotically (28 patients) and $22.6 \%$ (55 patients) laparoscopically with $65.9 \%$ (160 patients) via laparotomy.

There were no significant differences in any of the preoperative parameters studied [Table 2]. Tumors were evenly distributed between the two groups with 12 segmentectomies in the CR group comprising seven tumors in the anterior segments and five in the deep segments. A right or left hepatectomy was required in four patients, with nine left lateral segmentectomies being done in the CR group. This compares to segmentectomies being done in 23 patients in the HR group, with 16 in the anterior segments and 7 in the deep segments. A right hepatectomy was required in 16 patients, a left hepatectomy in 5 patients, and left lateral hepatectomies were done in 5 patients in the HR group. The lesions tended to be in the deeper segments in the HR group, but this did not achieve statistical significance $(P=0.064)$. The HR group tended to do more major resections, $42.9 \%$ vs. $21.4 \%$, but this was not statistically significant $(P=0.08)$ [Table 3 ]. 
Table 1. Distribution of liver resection for colorectal liver metastases by the handheld robotic (HR) group compared to the complete robotic (CR) group done with the complete robotic surgical system. Cases are divided into open, laparoscopic, and robotic cases

\begin{tabular}{lllllll}
\hline & Open & Lap & Robotic & Overall MIS & Total & P-value \\
\hline Hand-held Robotic Group (HR) (\%) & $4(7.5)$ & 0 & $49(92.5)$ & $49(92.5)$ & 53 & $<\mathbf{0 . 0 0 1}$ \\
Complete Robotic Group (CR) (\%) & $160(65.9)$ & $55(22.6)$ & $28(11.5)$ & $83(34.2)$ & 243 & \\
\hline
\end{tabular}

Table 2. Preoperative characteristics of patients undergoing liver resection for colorectal liver metastases with either handheld robotics (HR) or complete robotics (CR)

\begin{tabular}{llll}
\hline & $\begin{array}{l}\text { Handheld robotics (HR) } \\
\boldsymbol{n = 4 9}\end{array}$ & $\begin{array}{l}\text { Complete robotics (CR) } \\
\boldsymbol{n}=\mathbf{2 8}\end{array}$ & P-value \\
\hline Mean age (range) & $63.0(32-93)$ & $60.8(41-83)$ & 0.226 \\
Male:Female & $29: 20$ & $15: 13$ & 0.641 \\
ASA (\%) & $1=1$ & $1=1$ & 0.918 \\
& $2=20$ & $2=11$ & \\
& $3=28$ & $3=16$ & 0.254 \\
BMI kg/m (range) $^{2}$ & $4=0$ & $26.7(19.4-39.0)$ & 1 \\
Neoadjuvant chemotherapy (\%) & $28.7(19.0-45.0)$ & $19(67.9)$ & 0.206 \\
Previous surgery & $34(69.4)$ & $16(57.1)$ & 0.134 \\
Cirrhosis (\%) & $36(73.5)$ & $3(10.7 \%)$ & 1 \\
PVE & $1(2.0 \%)$ & 0 & \\
\hline
\end{tabular}

ASA: Anesthesiology Society of America status; BMI: body mass index; PVE: portal vein embolization.

Table 3. Liver-specific procedure and intraoperative results of patients undergoing liver resection for colorectal metastases with either handheld robotics (HR) or complete robotics (CR)

\begin{tabular}{llll}
\hline Procedure & $\begin{array}{l}\text { Handheld robotics (HR) } \\
\boldsymbol{n}=\mathbf{4 9}\end{array}$ & $\begin{array}{l}\text { Complete robotics (CR) } \\
\boldsymbol{n}=\mathbf{2 8}\end{array}$ & P-value \\
\hline Major resection (\%) & $21(42.9)$ & $6(21.4)$ & 0.082 \\
Minor resection (\%) & $28(57.1)$ & $22(78.6)$ & 0.550 \\
Conversion (\%) & $1(2.0)$ & $2(7.1)$ & 0.764 \\
Mean EBL mL (range) & $264.3(30-3000)$ & $226.9(0-850)$ & 0.550 \\
Transfusions (\%) & $1(2.0 \%)$ & $2(7.1)$ & $\mathbf{0 . 0 4 0}$ \\
Mean OR time (range) & $234.4(60-386)$ & $289.5(107-522)$ & $\mathbf{0 . 0 0 1}$ \\
Pringle maneuver (\%) & $2(4.1)$ & $9(32.1)$ & \\
\hline
\end{tabular}

EBL: Estimated blood loss; OR: operating room.

The Pringle maneuver was used significantly more in the CR group, $4.1 \%$ vs. $32.1 \%$, respectively $(P<0.001)$, but the mean estimated blood was not significantly different in either group, $264 \mathrm{~mL}$ (HR) vs. $227 \mathrm{~mL}$ (CR), respectively $(P>0.05)$ [Table 3]. The size and number of tumors removed were not significantly different between the groups, but the HR group had significantly more negative resection margins, $100 \%$ vs. $89.3 \%$, respectively $(P=0.049)$, and a significantly larger resection margin, $9.6 \mathrm{~mm} v s .6 .7 \mathrm{~mm}$, respectively $(P=$ 0.008). Although the length of stay tended to be longer after CR hepatectomy, 4.9 days vs. 6.8 days, this was not statistically significant $(P=0.089)$. Overall survival at one and two years and median survival were $100 \%$, 89.4\%, and 39 months after HR vs. 100\%, 72.9\%, and 34 months after $C R$, respectively $(P=0.055)$ [Figure 3 ]. Disease-free survival at one and two years and median survival equaled $75.7 \%, 48.2 \%$. and 23 months $v s$. $70.1 \%, 22.6 \%$. and 18 months after HR and CR, respectively $(P=0.403)$ [Figure 4]. 


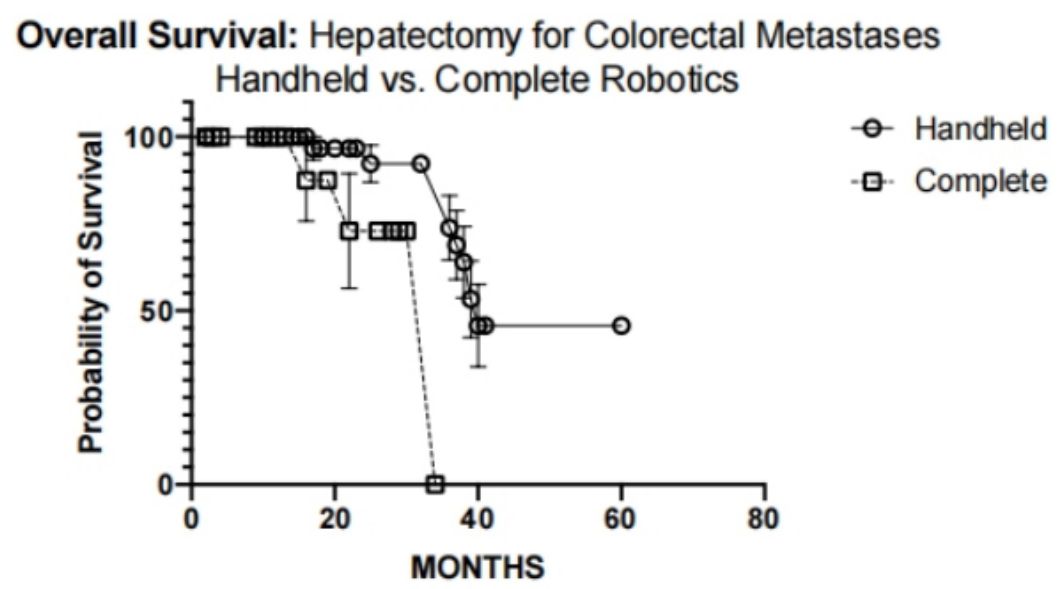

Figure 3. Overall survival of patients undergoing liver resection for colorectal metastases with either handheld robotics (HR) or complete robotics (CR).

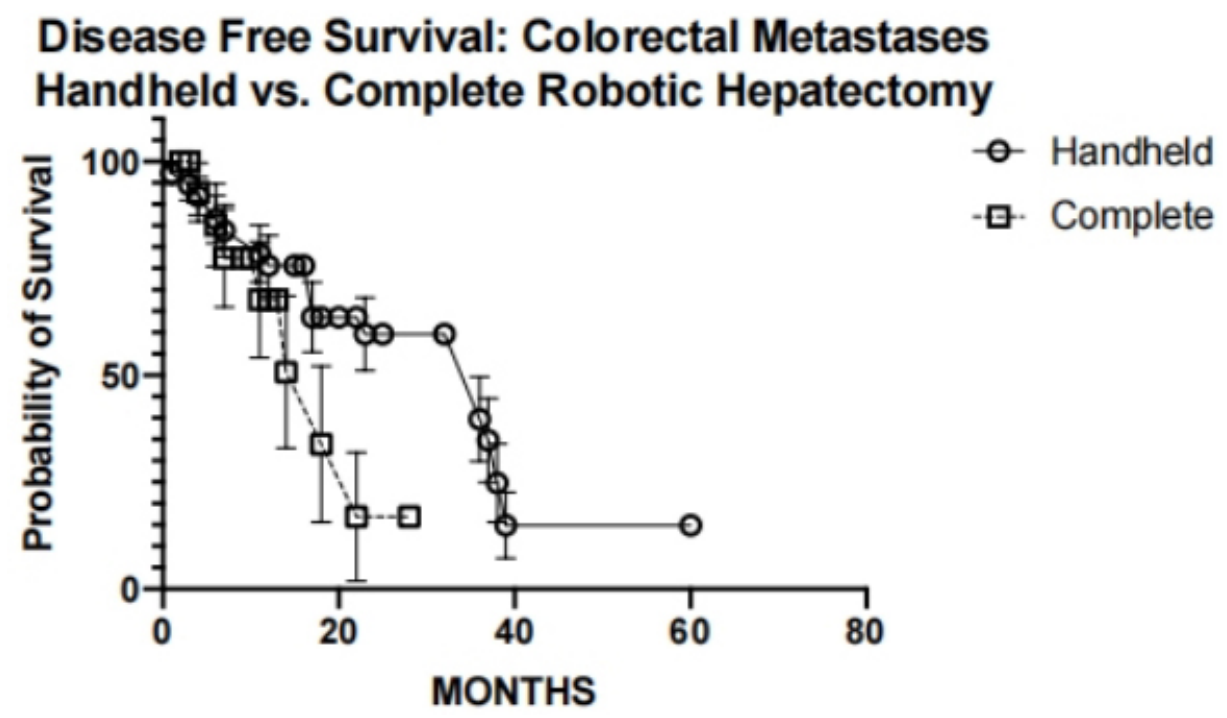

Figure 4. Overall survival of patients undergoing liver resection for colorectal metastases with either handheld robotics (HR) or complete robotics (CR).

\section{DISCUSSION}

When we compared cases done with HR $v$ s. CR, similar to other studies, we found that cases done with the CR approach were statistically longer than after HR [Table 3], but estimated blood loss was not higher as in most studies ${ }^{[35]}$. It is unclear if more complex liver resections will one day be done by the CR approach as suggested by this German study ${ }^{[36]}$, but what is clear is that whatever approach a surgeon is most comfortable with is clearly better for outcomes in the short term ${ }^{[37]}$. Conversion, morbidity, mortality, and reoperation rates were similar between the two groups and well within the reported literature ranges $[\text { Table } 4]^{[38]}$. The Ro resection rate and margin distance were statistically better in the HR group (all $P<$ 0.05), but this could be explained by the fact that the results from the CR group are rather early in their experience, with only 28 cases of CR hepatectomy at three centers compared to almost twice that by a single surgeon [Table 5]. The issue of whether laparoscopic or CR approaches have superior resection margins is currently unknown, with conflicting results ${ }^{[39]}$. Notably, although LOS tended to be shorter after HR, this was not statistically significant when compared to CR resections $(P=0.09)$ [Table 5]. An analysis of the 
Table 4. Postoperative morbidity and mortality of patients undergoing liver resection for colorectal metastases with either handheld robotics (HR) or complete robotics (CR)

\begin{tabular}{llll}
\hline & $\begin{array}{l}\text { Handheld robotics (HR) } \\
\boldsymbol{n = 4 9}\end{array}$ & $\begin{array}{l}\text { Complete robotics (CR) } \\
\boldsymbol{n = 2 8}\end{array}$ & P-value \\
\hline Mortality 30-day (\%) & $1(2.0)$ & 0 & 1 \\
Mortality 90-day (\%) & $2(4.1)$ & 0 & 0.531 \\
Re-operation & $1(2.0)$ & 0 & 1 \\
Dindo-clavien 30-day & & 0 & 1 \\
Bile leak & $1^{\star}(2.0)$ & $1(3.6)$ & 0.372 \\
I-II & 0 & $2(7.1)$ & 0.619 \\
III-V (\%) & $2^{\star}(4.1)$ & $3(10.7)$ & 0.347 \\
All (\%) & $2(4.1)$ & & \\
\hline
\end{tabular}

${ }^{\star}$ Denotes the same patient.

Table 5. Postoperative outcomes and pathological characteristics of patients undergoing liver resection for colorectal metastases with either handheld robotics (HR) or complete robotics (CR)

\begin{tabular}{llll}
\hline Tumor size (cm) (range) & $3.0(1.0-5.0)$ & $2.5(0.6-4.5)$ & 0.054 \\
No. of tumors (range) & $1.3(1-3)$ & $1.4(1-3)$ & 0.509 \\
Resection & & $25(89.3)$ & $\mathbf{0 . 0 4 9}$ \\
R0 (\%) & $49(100)$ & $3(10.7)$ & \\
R1 & 0 & 0 & $\mathbf{0 . 0 0 8}$ \\
R2 & 0 & $6.7(0-40)$ & 0.089 \\
Resection margin (mm) (range) & $9.6(1-30)$ & $6.8(0-23)$ & \\
Mean LOS (range) & $4.9(1-17)$ & & \\
\hline
\end{tabular}

LOS: Length of stay.

learning curve of these patients done via handheld robotic techniques has already been published and found better learning curves when surgeons undergo formal training for these techniques before the initiation of their independent careers ${ }^{[38]}$.

MI liver resection has numerous potential advantages ranging from decreased blood loss and hospital stay in the short term to potentially improved survival in the long term ${ }^{[25]}$. The reality is, however, that only highly selected cases are removed with MI techniques. At the same time, focus has shifted to the utilization of CRSS to see if one of these expensive platforms can have any real advantages for patients with pathologies such as CRLM. Although a survival benefit was suggested from the Kaplan-Meier curves, this was not highlighted due to the large differences in follow-up between the two groups, and we simply made the assumption that, at worst, the HR and CR group had similar overall and disease-free survival.

What interested us the most was why and how could the vast majority of cases in the HR group be done minimally invasively [Table 1], and this led us to the most obvious conclusion: haptics. Most robotic surgeons who use the complete robotic system use visual cues and, in general, do not believe that haptics are necessary. On the contrary, surgeons who do not use the CR system state that the lack of haptics is one of the main reasons for not adopting this technology. More recently, surgeons have begun to realize that some devices already perform some autonomous actions and that the exponential growth of AI will only enhance the growth and evolution of more autonomous surgical actions ${ }^{[28]}$. Perhaps more importantly, our findings 
suggest that there is an inherent contradiction between the CRSS and haptics. If the logical ultimate goal of the CRSS is increasing autonomy, is haptics going to ultimately really be that important ${ }^{[2]}$ ?

An excellent review article was recently published by a group of engineers that touched upon these points and also questioned the ultimate goal of increasing autonomy in surgery ${ }^{[40]}$. The authors expressed concern with the all or nothing attitude regarding autonomy in surgery and noted a concerning lack of literature regarding this topic. They specifically questioned why current trends in robotic development are focused on CRSS, and how it might be best if the surgeon were "kept in the loop" and not excluded from the equation $^{[40]}$. Perhaps the best way to accomplish this would be through handheld robotic instruments (Jaimy, Endocontrol, Grenoble, France) [Figure 5].

However, the question remains: why was the HR surgeon able to do the vast majority of cases minimally invasively? There is little question that a robotically controlled laparoscope holder that provides a steady image can decrease eye fatigue and potentially enable MI surgeons to do more complex procedures because of the increased control. The addition of having an assistant with two hands instead of one has obvious potential advantages, but the decreased fatigue of this assistant also needs to be taken into account. Once commercially available AI autonomous laparoscope tracking is available, this will also decrease stress and fatigue of the operating team. The potential advantages of operating on a completely stable image also has potential benefits in decreased inadvertent intra-abdominal injury with the possibility of decreased intraoperative and postoperative complications.

The robotically controlled GIA staplers also have the ability to decrease stress to the surgeon and delivery of a more precise deployment of the staple lines. The sensor on the device will also prevent stapling of tissue that is too thick, potentially reducing complications such as bleeding and bile leak. Conversely, this technology could also be preventing the stapling of tissue that is too thin, similarly decreasing the risk of intraoperative and postoperative complications. In a recent animal study, powered stapled anastomoses were found to have a higher burst pressure when compared to conventional stapling techniques ${ }^{[32,33,41]}$. On the surface, these advantages may appear to be minor benefits; nonetheless, our data clearly show that a potential benefit to HR liver resection in increasing the number of resections that can be done MI may exist, and this at least warrants further exploration. Although not utilized in our study, another potential advantage to the HR approach is the ability to do hand-assisted approaches. Many robotic surgeons will state that they do not need to utilize hand assistance, and in carefully selected cases we would also agree, but if the utilization of this technique enables more patients to undergo MI liver resection why would one not use this option ${ }^{[42]}$ ?

What about other examples of handheld "smart" devices or examples of weak AI? As opposed to strong AI where the device acts more independently, weak AI has limited sensing ability and limited autonomy. The above-mentioned handheld devices are good examples of weak AI. Several other interventional and surgical specialties have begun using intelligent handheld robotic instruments with weak AI. Automated venous puncture devices using robotics, ultrasound, and computer vision (CV) have been developed that show similar success rates as with humans ${ }^{[43]}$. In addition to ultrasound, acoustic waves have also been harnessed to give MI surgeons the ability to perceive different tissues, and they may be utilized to permit improved surgical dissection ${ }^{[4]}$. Recent exponential advances in machine learning, deep learning, and CV make this field of robotics particularly interesting for dissection and again raise concerns that our obsession with haptics may be ill-advised and that many other avenues of AI should also be explored ${ }^{[45]}$. 


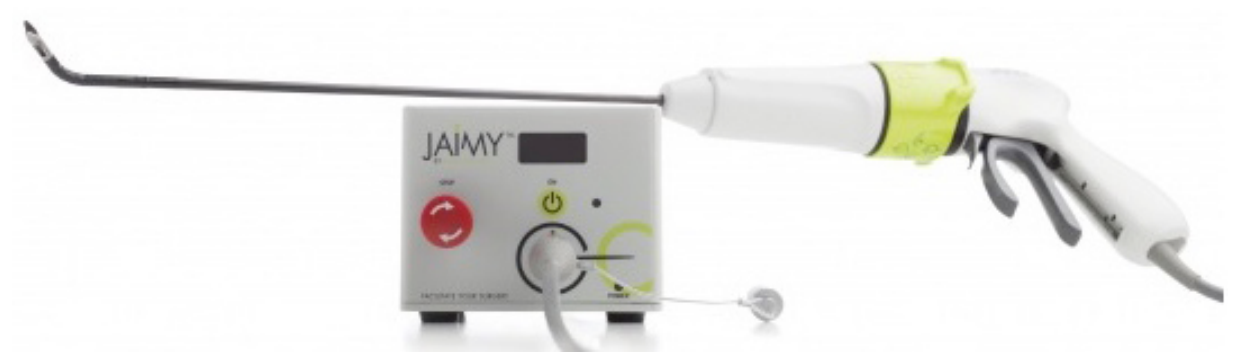

Figure 5. Robotically controlled handheld surgical dissector/needle driver (Jaimy, Endocontrol, Grenoble, France).

Tremor stabilizing devices have been created for eye surgery ${ }^{[46,47]}$. As mentioned, complete robotic systems already have the ability to provide the surgeon haptics and have some form of tremor dampening; however, due to the need for this dampening, haptics in their current form are bothersome, and this feature is essentially unusable. The problems are that a filter needs to be used to compensate for the physiological resting tremor that the surgeons have and the phase delay inherent in sensors; their interaction with the filtering software is too slow with current technology; and the time that it takes to interpret the data delays the reaction time too much to make this a viable form of feedback ${ }^{[48]}$.

Notably, haptics with force feedback have been shown to improve surgical trainees when they learn MI techniques on simulators using virtual reality (VR), and they are believed to reduce surgical errors and potentially the rate of complications ${ }^{[49]}$. Researchers have actually developed techniques to account for tremor and use predictive movement algorithms to provide some form of haptics for surgeons using technology that was originally devised to intervene in cases of cyberattacks during surgery, but this technology has not yet been validated in patient ${ }^{[50]}$. Some groups have begun using a bimodal vibrotactile system that uses AI to give surgeons information feedback on structures that they are palpating using a specialized sensor. As opposed to haptics while using the actual surgical instruments, the idea is to use a sensing probe during part of the operation to help with tissue identification ${ }^{[51]}$. Although an interesting work around, switching instruments for a sensing probe and then back to surgical instruments will certainly add time to operations.

Groups trying to provide haptics of robotic surgical instruments have been able to create some form of haptics, but only based on estimations. In this scenario, a force sensor is not used at the end effector of the surgical instrument, but an estimation of forces is done by calculating from the tension of the wires in the robotic instruments. When trainees were given visual cues and this type of haptics, they preferred it to using only visual cues during surgical maneuvers. Although interesting, it is too early to tell if an estimation of haptics alone will be enough during actual surgery ${ }^{[52]}$.

HR devices with haptics have been developed for eye surgery using fiber Bragg grating sensors in optical fibers. These devices are able to detect and perceive forces from three degrees of freedom. Unlike laparoscopic surgery, microsurgery involves forces that are imperceptible to humans, and because of this the learning curve for eye surgery can be much longer, taking up to 10 years to master certain procedures. Forces only measure $1 \mathrm{~N}$ in eye surgery, which is several folds less than that seen in laparoscopy, and when using this technology researchers were able to measure down to forces of a millinewton ${ }^{[53]}$. This perhaps explains why eye surgeons have embraced HR more so than general surgeons. 
Methods to improve surgical gestures such as tracing in eye microsurgery have also been developed using active handheld manipulators, and they have the potential to improve surgical dissection itself ${ }^{[54]}$. Additional benefits of HR also include decreased cost and improved workflow times due to the absence of significant setup times ${ }^{[3,55]}$. Fortunately, some groups have begun developing HR surgical dissecting instruments, as well as studying them in VR simulators ${ }^{[56-58]}$.

Other examples of intelligent HR instruments can be found in interventional radiology, orthopedics, neurosurgery, and endoscopy. Automatic guidance systems have been developed for percutaneous needle placements to improve biopsies and interventional procedures such as treatment of endo-leaks and for ablation $s^{[59]}$. Orthopedic surgeons have used HR for arthroplasty enabling better pin placement for years ${ }^{[0,61]}$. In neurosurgery, small robots have been attached to stereotaxic frames to permit superior computer automated aspiration of brain tissue in animal models, and HR has been used to perform craniotomy with submillimeter accuracy and a decreased risk of complications when compared to standard robots $^{[62]}$. Technology has even been developed to give haptic feedback during flexible endoscopy ${ }^{[63]}$.

The innovation seen in other surgical and interventional disciplines begs the question: why is there not more of an emphasis on researching handheld robotic or artificially intelligent devices or pushing them in general surgery? With such an emphasis being placed on the CRSS one wonders if hospital systems will have the economic ability to purchase these smaller robots. Furthermore, most hospitals around the world cannot afford the CRSS, this is effectively creating a 2-tiered system where most poorer hospitals cannot afford robotics. Notably, the latest da Vinci Xi system costs approximately 2 million dollars while the ViKY robotically controlled laparoscope holder costs closer to $\$ 60,000$. Possibly by diverging research dollars into smaller HR we can enable more people to benefit from devices enhanced by computers and AI.

\section{Conclusion}

Whether one type of the MI approach used is superior to another will be answered as more centers gain expertise with laparoscopic and robotic liver resection. HR has the potential advantage of maintaining haptics, which could increase the rate of patients who can undergo MI hepatectomy, with the added benefit of being more cost-effective and accessible. A potential drawback of HR is the size constraints limiting technological advancements. Regardless, HR may enable surgeons to stay in the loop by maintaining haptics and result in the development of autonomous actions in surgery more safely and ultimately more quickly than via CRSS. It is unclear whether the CR approach will ever need haptics, especially since the CR system may evolve to autonomous surgery with the robot itself being the device that needs to perceive haptics and not the operating surgeon.

\section{DECLARATIONS}

\section{Authors' contributions}

Drafting of manuscript and editing: Gumbs AA, Croner R

Collecting of data, statistics and interpretation: Gumbs AA, Abu-Hilal M, Tsai TJ, Starker L, Chouillard E, Croner R

Editing and provided administrative support: Gumbs AA, Abu-Hilal M, Tsai TJ, Starker L, Chouillard E, Croner R

\section{Availability of data and materials}

Data will be made available upon reasonable request. 


\section{Financial support and sponsorship}

None.

\section{Conflicts of interest}

All authors declared that there are no conflicts of interest.

\section{Ethical approval and consent to participate}

Retrospective study, informed consent obtained on all patients prior to surgery.

\section{Consent for publication}

Not applicable.

\section{Copyright}

(c) The Author(s) 2021.

\section{REFERENCES}

1. Giulianotti PC, Coratti A, Angelini M, et al. Robotics in general surgery: personal experience in a large community hospital. Arch Surg 2003;138:777-84. DOI PubMed

2. Vibert E, Denet C, Gayet B. Major digestive surgery using a remote-controlled robot: the next revolution. Arch Surg 2003;138:1002-6. DOI PubMed

3. Milone M, Manigrasso M, Velotti N, et al. Completeness of total mesorectum excision of laparoscopic versus robotic surgery: a review with a meta-analysis. Int $J$ Colorectal Dis 2019;34:983-91. DOI PubMed

4. Unger SW, Unger HM, Bass RT. AESOP robotic arm. Surg Endosc 1994;8:1131. DOI PubMed

5. Kraft BM, Jäger C, Kraft K, Leibl BJ, Bittner R. The AESOP robot system in laparoscopic surgery: increased risk or advantage for surgeon and patient? Surg Endosc 2004;18:1216-23. DOI PubMed

6. Gagner M. Laparoscopic partial hepatectomy for liver tumor. Surg Endosc 1992;6:97-8. DOI

7. Abu Hilal M, McPhail MJ, Zeidan B, et al. Laparoscopic versus open left lateral hepatic sectionectomy: a comparative study. Eur J Surg Oncol 2008;34:1285-8. DOI PubMed

8. Hilal M, Pearce NW. Laparoscopic left lateral liver sectionectomy: a safe, efficient, reproducible technique. Dig Surg 2008;25:305-8. DOI PubMed

9. McPhail MJ, Scibelli T, Abdelaziz M, Titi A, Pearce NW, Abu Hilal M. Laparoscopic versus open left lateral hepatectomy. Expert Rev Gastroenterol Hepatol 2009;3:345-51. DOI PubMed

10. Gayet B, Cavaliere D, Vibert E, et al. Totally laparoscopic right hepatectomy. Am J Surg 2007;194:685-9. DOI PubMed

11. Cherqui D, Belghiti J. [Hepatic surgery. What progress? What future?]. Gastroenterol Clin Biol 2009;33:896-902. DOI PubMed

12. Viganò L, Tayar C, Laurent A, Cherqui D. Laparoscopic liver resection: a systematic review. J Hepatobiliary Pancreat Surg 2009;16:410-21. DOI PubMed

13. Gumbs AA, Leventhal A, Hoffman JP. Video: laparoscopic right hepatectomy and partial resection of the diaphragm for liver metastases. Surg Endosc 2011;25:3441-3. DOI PubMed

14. Tsai TJ, Chouillard EK, Gumbs AA. Laparoscopic right hepatectomy with intrahepatic transection of the right bile duct. Ann Surg Oncol 2012;19:467-8. DOI PubMed

15. Kluger MD, Vigano L, Barroso R, Cherqui D. The learning curve in laparoscopic major liver resection. J Hepatobiliary Pancreat Sci 2013;20:131-6. DOI PubMed

16. Dagher I, Gayet B, Tzanis D, et al. International experience for laparoscopic major liver resection. J Hepatobiliary Pancreat Sci 2014;21:732-6. DOI PubMed

17. Wakabayashi G, Cherqui D, Geller DA, et al. Recommendations for laparoscopic liver resection: a report from the second international consensus conference held in Morioka. Ann Surg 2015;261:619-29. DOI PubMed

18. Gumbs AA, Dhamija A, Gorman D, Ben-menachem T. Laparoscopic right hepatectomy, common bile duct excision, and left hepaticojejunostomy for biliary tract: intraductal papillary mucinous tumor. Videoscopy 2018:28. DOI

19. Abu Hilal M, Aldrighetti L, Dagher I, et al. The southampton consensus guidelines for laparoscopic liver surgery: from indication to implementation. Ann Surg 2018;268:11-8. DOI PubMed

20. Berkelman P, Cinquin P, Boidard E, Troccaz J, Létoublon C, Long JA. Development and testing of a compact endoscope manipulator for minimally invasive surgery. Comput Aided Surg 2005;10:1-13. DOI PubMed

21. Gumbs AA, Crovari F, Vidal C, Henri P, Gayet B. Modified robotic lightweight endoscope (ViKY) validation in vivo in a porcine model. Surg Innov 2007;14:261-4. DOI PubMed

22. Gumbs AA, Tsai TJ, Hoffman JP. Initial experience with laparoscopic hepatic resection at a comprehensive cancer center. Surg Endosc 2012;26:480-7. DOI PubMed

23. Bensignor T, Morel G, Reversat D, Fuks D, Gayet B. Evaluation of the effect of a laparoscopic robotized needle holder on ergonomics 
and skills. Surg Endosc 2016;30:446-54. DOI PubMed

24. Ohdaira H, Noro T, Terada H, et al. New double-stapling technique for esophagojejunostomy and esophagogastrostomy in gastric cancer surgery, using a peroral intraluminal approach with a digital stapling system. Gastric Cancer 2009;12:101-5. DOI PubMed

25. Syn NL, Kabir T, Koh YX, et al. Survival advantage of laparoscopic versus open resection for colorectal liver metastases: a metaanalysis of individual patient data from randomized trials and propensity-score matched studies. Ann Surg 2020;272:253-65. DOI PubMed

26. Wang J. To use or not to use propensity score matching? Pharm Stat 2021;20:15-24. DOI PubMed

27. Gumbs AA, Frigerio I, Spolverato G, et al. Artificial intelligence surgery: how do we get to autonomous actions in surgery? Sensors (Basel) 2021;21:5526. DOI PubMed PMC

28. Gumbs AA, Perretta S, d'Allemagne B, Chouillard E. What is artificial intelligence surgery? Art Int Surg 2021;1:1-10. DOI

29. Gumbs AA, Bar-Zakai B, Gayet B. Totally laparoscopic extended left hepatectomy. J Gastrointest Surg 2008;12:1152. DOI PubMed

30. Gumbs AA, Gayet B. Multimedia article. Totally laparoscopic extended right hepatectomy. Surg Endosc 2008;22:2076-7. DOI PubMed

31. Gumbs AA, Gayet B. Video: the lateral laparoscopic approach to lesions in the posterior segments. J Gastrointest Surg 2008;12:1154. DOI PubMed

32. Gumbs AA, Gayet B. Adopting Gayet's techniques of totally laparoscopic liver surgery in the United States. Liver Cancer 2013;2:515. DOI PubMed PMC

33. Gumbs AA, Gayet B, Gagner M. Laparoscopic liver resection: when to use the laparoscopic stapler device. HPB (Oxford) 2008;10:296-303. DOI PubMed PMC

34. Dindo D, Demartines N, Clavien PA. Classification of surgical complications: a new proposal with evaluation in a cohort of 6336 patients and results of a survey. Ann Surg 2004;240:205-13. DOI PubMed PMC

35. Guan R, Chen Y, Yang K, et al. Clinical efficacy of robot-assisted versus laparoscopic liver resection: a meta analysis. Asian J Surg 2019;42:19-31. DOI PubMed

36. Rahimli M, Perrakis A, Schellerer V, et al. Robotic and laparoscopic liver surgery for colorectal liver metastases: an experience from a German Academic Center. World J Surg Oncol 2020;18:333. DOI PubMed PMC

37. Giulianotti PC, Coratti A, Sbrana F, et al. Robotic liver surgery: results for 70 resections. Surgery 2011;149:29-39. DOI PubMed

38. Gumbs AA, Hilal MA, Croner R, Gayet B, Chouillard E, Gagner M. The initiation, standardization and proficiency (ISP) phases of the learning curve for minimally invasive liver resection: comparison of a fellowship-trained surgeon with the pioneers and early adopters. Surg Endosc 2021;35:5268-78. DOI PubMed

39. Croner RS, Perrakis A, Brunner M, Matzel KE, Hohenberger W. Pioneering robotic liver surgery in germany: first experiences with liver malignancies. Front Surg 2015;2:18. DOI PubMed PMC

40. Battaglia E, Boehm J, Zheng Y, Jamieson AR, Gahan J, Majewicz Fey A. Rethinking autonomous surgery: focusing on enhancement over autonomy. Eur Urol Focus 2021;7:696-705. DOI PubMed

41. Sahloul M, Kapoulas S, Giet L, et al. Does powered stapler improve the mechanical integrity of gastrojejunal anastomosis compared to the current techniques? J Minim Access Surg 2021. DOI PubMed

42. Donisi G, Zerbi A. What role does hand-assistance have in minimally invasive pancreatic surgery? Mini-invasive Surg 2021;5:38. DOI

43. Leipheimer JM, Balter ML, Chen AI, et al. First-in-human evaluation of a hand-held automated venipuncture device for rapid venous blood draws. Technology (Singap World Sci) 2019;7:98-107. DOI PubMed PMC

44. Ostler D, Seibold M, Fuchtmann J, et al. Acoustic signal analysis of instrument-tissue interaction for minimally invasive interventions. Int J Comput Assist Radiol Surg 2020;15:771-9. DOI PubMed PMC

45. Ajani TS, Imoize AL, Atayero AA. An overview of machine learning within embedded and mobile devices-optimizations and applications. Sensors (Basel) 2021;21:4412. DOI PubMed PMC

46. Maclachlan RA, Becker BC, Tabarés JC, Podnar GW, Lobes LA Jr, Riviere CN. Micron: an actively stabilized handheld tool for microsurgery. IEEE Trans Robot 2012;28:195-212. DOI PubMed PMC

47. Song C, Gehlbach PL, Kang JU. Active tremor cancellation by a "smart" handheld vitreoretinal microsurgical tool using swept source optical coherence tomography. Opt Express 2012;20:23414-21. DOI PubMed PMC

48. Tatinati S, Wang Y, Shafiq G, Veluvolu KC. Online LS-SVM based multi-step prediction of physiological tremor for surgical robotics. Annu Int Conf IEEE Eng Med Biol Soc 2013;2013:6043-6. DOI PubMed

49. van der Meijden OA, Schijven MP. The value of haptic feedback in conventional and robot-assisted minimal invasive surgery and virtual reality training: a current review. Surg Endosc 2009;23:1180-90. DOI PubMed PMC

50. Li X, Kesavadas T. Surgical robot with environment reconstruction and force feedback. Annu Int Conf IEEE Eng Med Biol Soc 2018;2018:1861-6. DOI PubMed

51. Abiri A, Juo YY, Tao A, et al. Artificial palpation in robotic surgery using haptic feedback. Surg Endosc 2019;33:1252-9. DOI PubMed PMC

52. Saracino A, Oude-Vrielink TJC, Menciassi A, Sinibaldi E, Mylonas GP. Haptic intracorporeal palpation using a cable-driven parallel robot: a user study. IEEE Trans Biomed Eng 2020;67:3452-63. DOI PubMed

53. Wang Z, Wang S, Zuo S. A hand-held device with 3-DOF haptic feedback mechanism for microsurgery. Int J Med Robot 2019;15:e2025. DOI PubMed

54. Choi DY, Sandoval R, MacLachlan RA, Ho L, Lobes LA, Riviere CN. Test of tracing performance with an active handheld micromanipulator. Annu Int Conf IEEE Eng Med Biol Soc 2007;2007:3638-41. DOI PubMed 
55. Payne CJ, Yang GZ. Hand-held medical robots. Ann Biomed Eng 2014;42:1594-605. DOI PubMed

56. Zahraee AH, Szewczyk J, Morel G. Simulation for optimal design of hand-held surgical robots. Annu Int Conf IEEE Eng Med Biol Soc 2009;2009:270-3. DOI PubMed

57. Zahraee AH, Paik JK, Szewczyk J, Morel G. Toward the development of a hand-held surgical robot for laparoscopy. IEEE/ASME Trans Mechatron 2010. DOI

58. Pereira R, Moreira AHJ, Leite M, et al. Hand-held robotic device for laparoscopic surgery and training. 2014 IEEE 3 nd International Conference on Serious Games and Applications for Health (SeGAH); 2014 May 14-16; Rio de Janeiro, Brazil. IEEE; 2014. p. 1-8. DOI

59. Hiraki T, Kamegawa T, Matsuno T, Komaki T, Sakurai J, Kanazawa S. Zerobot $®$ : a remote-controlled robot for needle insertion in CT-guided Interventional radiology developed at okayama university. Acta Med Okayama 2018;72:539-46. DOI PubMed

60. Battenberg AK, Netravali NA, Lonner JH. A novel handheld robotic-assisted system for unicompartmental knee arthroplasty: surgical technique and early survivorship. J Robot Surg 2020;14:55-60. DOI PubMed PMC

61. Ma JH, Sefati S, Taylor RH, Armand M. An active steering hand-held robotic system for minimally invasive orthopaedic surgery using a continuum manipulator. IEEE Robot Autom Lett 2021;6:1622-9. DOI PubMed PMC

62. Liang B, Zhang L, Moffitt C, Li Y, Lin DT. An open-source automated surgical instrument for microendoscope implantation. $J$ Neurosci Methods 2019;311:83-8. DOI PubMed PMC

63. Lai W, Cao L, Tan RX, et al. An integrated sensor-model approach for haptic feedback of flexible endoscopic robots. Ann Biomed Eng 2020;48:342-56. DOI PubMed 